\title{
Electronic structure of the neutral silicon-vacancy center in diamond
}

\author{
B. L. Green, ${ }^{1,}{ }^{*}$ M. W. Doherty, ${ }^{2}$ E. Nako, ${ }^{1,3}$ N. B. Manson, ${ }^{2}$ U. F. S. D'Haenens-Johansson, ${ }^{4}$ \\ S. D. Williams, ${ }^{5}$ D. J. Twitchen, ${ }^{5}$ and M. E. Newton ${ }^{1,3}$ \\ ${ }^{1}$ Department of Physics, University of Warwick, Coventry CV4 7AL, United Kingdom \\ ${ }^{2}$ Laser Physics Centre, Research School of Physics and Engineering, Australian National University, \\ Australian Capital Territory 2601, Australia \\ ${ }^{3}$ EPSRC Centre for Doctoral Training in Diamond Science and Technology, Materials \& Analytical Sciences, \\ University of Warwick, Coventry CV4 7AL, United Kingdom \\ ${ }^{4}$ Gemological Institute of America, 50 West 47th Street, New York, New York 10036, USA \\ ${ }^{5}$ Element Six Limited, Global Innovation Centre, Fermi Avenue, Didcot OX11 OQR, United Kingdom
}

(Received 4 May 2018; revised manuscript received 3 September 2018; published 17 April 2019)

\begin{abstract}
The neutrally charged silicon vacancy in diamond is a promising system for quantum technologies that combines high-efficiency optical spin initialization with long spin lifetimes $\left(T_{2} \approx 1 \mathrm{~ms}\right.$ at $\left.4 \mathrm{~K}\right)$ and up to $90 \%$ of optical emission into its $946-\mathrm{nm}$ zero-phonon line. However, the electronic structure of $\mathrm{SiV}^{0}$ is poorly understood, making further exploitation difficult. Performing photoluminescence spectroscopy of $\mathrm{SiV}^{0}$ under uniaxial stress, we find the previous excited electronic structure of a single ${ }^{3} A_{1 u}$ state is incorrect, and identify instead a coupled ${ }^{3} E_{u}-{ }^{3} A_{2 u}$ system, the lower state of which has forbidden optical emission at zero stress and efficiently decreases the total emission of the defect. We propose a solution employing finite strain to define a spin-photon interface scheme using $\mathrm{SiV}^{0}$.
\end{abstract}

DOI: 10.1103/PhysRevB.99.161112

Optically accessible solid state defects are promising candidates for scalable quantum information processing [1,2]. Diamond is the host crystal for two of the most-studied point defects: the negatively charged nitrogen-vacancy $\left(\mathrm{NV}^{-}\right)$ center [3], and the negatively charged silicon-vacancy $\left(\mathrm{SiV}^{-}\right)$ center [4]. $\mathrm{NV}^{-}$has been successful in a broad range of fundamental [5,6] and applied [7-9] quantum experiments, with spin-photon [10] and spin-spin [11] entanglement protocols well established. The superior photonic performance of $\mathrm{SiV}^{-}$, with $>70 \%$ of photonic emission into its zero-phonon line (ZPL), and insensitivity to electric fields yielding low spectral diffusion as a result of the defect's inversion symmetry, has enabled it to make a rapid impact in photonic quantum platforms $[12,13]$. However, $\mathrm{SiV}^{-}$possesses poor spin coherence lifetimes due to phononic interactions in the ground state [14], requiring temperatures of $<100 \mathrm{mK}$ to achieve a spin lifetime of $T_{2} \approx 13 \mathrm{~ms}$ with decoupling [15].

Recent work on $\mathrm{SiV}^{0}$, the neutrally charged silicon vacancy in diamond, has demonstrated that it combines high-efficiency optical spin polarization [16,17] with long spin lifetimes ( $T_{2} \approx 250 \mathrm{~ms}$ at $15 \mathrm{~K}$ with dynamical decoupling [16]) and a high Debye-Waller factor [16]: The defect potentially possesses the ideal combination of $\mathrm{SiV}^{-}$and $\mathrm{NV}^{-}$properties. Exploitation of these promising properties is hindered by a poor understanding of the defect's electronic structure. Electron paramagnetic measurements (EPR) of $\mathrm{SiV}^{0}$ indicate it has a spin-triplet ${ }^{3} A_{2 g}$ ground state and $D_{3 d}$ symmetry [18], with the silicon atom residing on axis in a split-vacancy configuration (Fig 1, inset). Optically excited EPR measurements

\footnotetext{
*b.green@warwick.ac.uk
}

directly relate the $\mathrm{SiV}^{0}$ spin system to a zero-phonon line (ZPL) at $946 \mathrm{~nm}$ [17]: Optical absorption experiments and density functional theory (DFT) calculations have assigned the ZPL excited state to ${ }^{3} A_{1 u}$ symmetry $[19,20]$. Temperaturedependent PL measurements indicate the presence of an optically inactive state below the luminescent excited state [19]. No optically detected magnetic resonance (ODMR) of $\mathrm{SiV}^{0}$ has been reported.

The advances in exploitation of $\mathrm{NV}^{-}$and $\mathrm{SiV}^{-}$have been driven by a concerted effort in the fundamental understanding of the physics of the centers themselves. In this Rapid Communication, we employ photoluminescence (PL) spectroscopy to study an ensemble of $\mathrm{SiV}^{0}$ under applied uniaxial stress, and show that the previous assignment of a single excited state ${ }^{3} A_{1 u}$ is incorrect. We find that the $946-\mathrm{nm}$ excited state is ${ }^{3} E_{u}$, with a ${ }^{3} A_{2 u}$ state approximately $6.8 \mathrm{meV}$ below it. The latter transition is forbidden by symmetry at zero stress and therefore efficiently reduces the emission intensity of unstrained $\mathrm{SiV}^{0}$ centers at low temperature. We propose a solution using engineered strain devices which enables an optically initializable spin-photon scheme with optical spin readout between the ${ }^{3} A_{2 g}$ ground state and ${ }^{3} A_{2 u}$ excited state. The latter state is shown definitively to participate in the optical spin polarization mechanism of $\mathrm{SiV}^{0}$, and opens a route to ODMR using $\mathrm{SiV}^{0}$. Finally, we demonstrate that the 976-nm transition associated with $\mathrm{SiV}^{0}$ [17], previously hypothesized to be a strain-induced transition [20], is actually a pseudolocal vibrational model (LVM) of $\mathrm{SiV}^{0}$ primarily involving the silicon atom.

We apply uniaxial stress to a diamond crystal grown by chemical vapor deposition: The crystal was intentionally doped during growth with ${ }^{29} \mathrm{Si}$-enriched silane to create $\mathrm{SiV}^{-}$ 

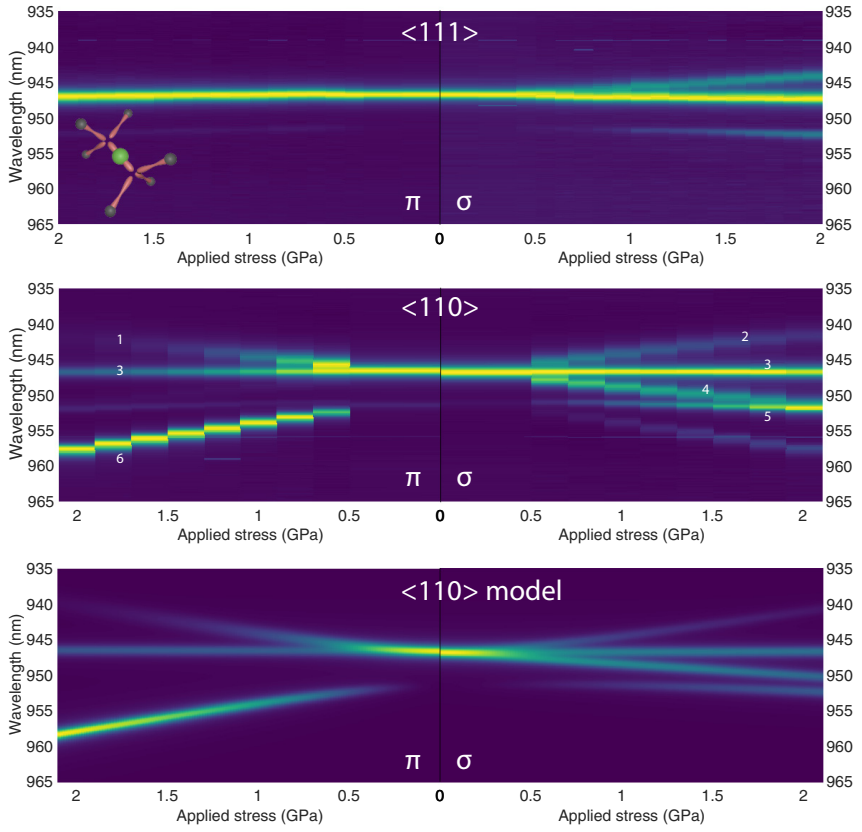

FIG. 1. $\mathrm{SiV}^{0}$ photoluminescence spectra at $80 \mathrm{~K}$ as a function of applied stress along $\langle 111\rangle$ (top) and $\langle 110\rangle$ (middle). In each case, $\pi(\sigma)$ indicates detection polarization parallel (perpendicular) to the stress direction. The transition at $946 \mathrm{~nm}$ splits into components $1-4$ under $\langle 110\rangle$ stress, with thermalization between the components observed at high stress indicating electronic degeneracy. A pair of stress-induced transitions $(5,6)$ originate at approximately $951 \mathrm{~nm}$. Inset, top: The geometric form of $\mathrm{SiV}^{0}$, with the $\mathrm{Si}$ atom on axis in the split-vacancy configuration. Bottom: Simulation of the $\langle 110\rangle$ stress spectra using the model described in the main text.

and $\mathrm{SiV}^{0}$ [see Supplemental Material (SM) for details [21]]. Uniaxial stress was applied to the sample using a home-built ram driven by pressurized nitrogen gas. PL measurements were collected under excitation at $785 \mathrm{~nm}$ as a function of applied stress in both the $\langle 111\rangle$ and $\langle 110\rangle$ directions [21]. We measured spectra for all four combinations of excitation and detection polarization parallel $(\pi)$ and perpendicular $(\sigma)$ to the stress axis. We find that the spectra are essentially invariant to the excitation polarization [21]. Our 1.58-nm (785-nm) excitation laser is above the $1.50-\mathrm{eV}$ ionization threshold for $\mathrm{SiV}^{0}[22]$ and therefore we believe we are creating the excited state of $\mathrm{SiV}^{0}$ via photoionization of $\mathrm{SiV}^{-}$as described in Ref. [19]. We thus focus on analyzing just the spectra for the two detection polarizations $(\pi, \sigma)$ arising from a single excitation polarization $(\pi)$.

The problem of uniaxial stress applied to a trigonal defect in a cubic crystal has been described several times [23-25], so we summarize the results for transitions to an orbital singlet ground state, as found in $\mathrm{SiV}^{0}$. In both $\langle 111\rangle$ and $\langle 110\rangle$ applied stress, the orientational degeneracy of the defect is lifted into two classes of orientation, classified by the angle between their high-symmetry axis and the uniaxial stress axis. For an orbital singlet-to-singlet $(A \leftrightarrow A)$ transition, only one transition per orientation is possible: When taking into account both orientation classes, we expect a maximum of two transitions per spectrum. In the orbital singlet-to-doublet $(A \leftrightarrow E)$ case, two transitions per orientation are possible, leading to a maximum of four transitions per spectrum. $\langle 111\rangle$ stress does not remove the electronic degeneracy of the $E_{x}, E_{y}$ orbitals for the orientation parallel to the applied stress, and hence a maximum of three transitions are expected.

For uniaxial stress applied to the $\langle 111\rangle$ axis, the 946-nm ZPL splits into three transitions, two of which are almost degenerate but which possess different emission polarization (Fig. 1). This is consistent with the $A \leftrightarrow E$ case described earlier. Under $\langle 110\rangle$ uniaxial stress, we identify four distinct components originating at the ZPL, again consistent with an $A \leftrightarrow E$ transition. The intensities of the different components varies as a function of applied stress, confirming the presence of electronic degeneracy in the excited state. For both stress directions, we observe additional lower-energy transitions originating at $\approx 951 \mathrm{~nm}$. The transitions gain intensity as a function of stress (Fig. 1). We measure only two components, indicating the presence of an additional orbital singlet state. At a constant applied stress of $\sigma_{\langle 110\rangle}=1.3 \mathrm{GPa}$, decreasing the temperature increases the intensity of the stress-induced transitions at the expense of the 946-nm transitions [Fig. 2(b)]. Therefore, we conclude the additional $A$ state lies close in energy to the excited $E$ state, rather than the ground ${ }^{3} A_{2 g}$.

To construct a model of the excited state behavior, we must establish the origin of the lower-energy $A$ state. There are possibilities: spin-orbit (SO) fine structure arising from the $E$ level; Jahn-Teller (JT) vibronic structure arising from the $E$ level; and a totally independent $A$ level. An SO interaction of $6.5 \mathrm{meV}(\approx 1.57 \mathrm{THz})$ is inconsistent with the SO magnitude in $\mathrm{SiV}^{-}\left(250 \mathrm{GHz}\right.$ [26]) and $\mathrm{GeV}^{-}(1.06 \mathrm{THz}$ [27]) and would yield additional $A$ and $E$ states (as in the $\mathrm{NV}^{-}$excited state [28]) and hence we reject this possibility. A JT distortion would place the $A$ state above the $E$ and hence is inconsistent with experiment. Additionally, the piezospectroscopic parameters describing the singlet and doublet states are significantly different [21], as would be expected if they arise from distinct electronic states [29]. We conclude that the singlet is an additional electronic state and is not derived from the doublet. Experimentally, we find the singlet transitions are polarized in pure $\sigma$ for $\langle 111\rangle$ stress, and pure $\sigma, \pi$ for $\langle 110\rangle$ stress (Fig. 1): This identifies the $A$ level as possessing $\Gamma_{2}^{-}$symmetry in the lowered $C_{2 h}$ symmetry of the defect under stress [21].

Building on previous numerical descriptions of a coupled $E-A$ system in trigonal symmetry [29], we construct a full analytical treatment of this problem [21]. The result of a leastsquares fit of this model simultaneously to the experimental $\langle 110\rangle$ and $\langle 111\rangle$ spectra as a function of stress is given in Fig. 2(a): Piezospectroscopic parameters are detailed in the SM [21]. The output of the model was tested by comparing it to the transition intensities of spectra measured as a function of temperature at a fixed $\sigma_{\langle 110\rangle}=1.3 \mathrm{GPa}$ [Fig. 2(b)]. The ordering and behavior of all transitions matches the experiment and hence we accept the coupled $E-A$ model as a suitable description of the $\mathrm{SiV}^{0}$ excited state.

There are several reasons why the model fit is not perfect. Intrinsic inhomogeneous stress will introduce nonlinearities into the line shifts at low stress; small misalignments or nonuniaxial stress will modify the shift rates from those taken into account by the model, which will be exacerbated if these effects are different in the two stress directions. Finally, Jahn-Teller interactions in the $E$ state, and pseudo-Jahn Teller 

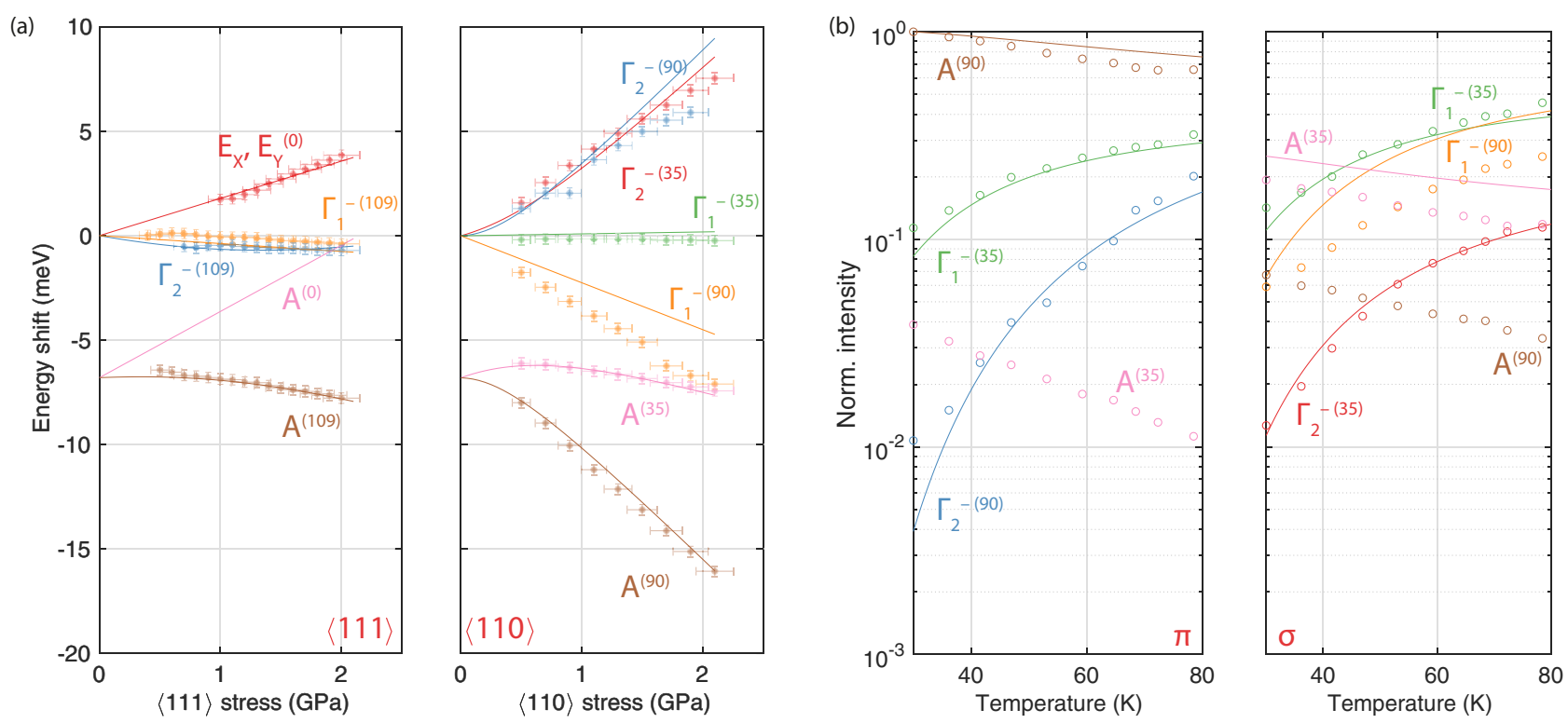

FIG. 2. Comparison of experiment (dots) with the coupled $E$ - $A$ model (lines). Transitions are labeled with the state $\left(A\right.$; and $\left\{\Gamma_{1}^{-}, \Gamma_{2}^{-}\right\}$for the $E$ state-see Ref. [21] for details) and the angle between the symmetry axis of the subensemble and the stress axis (in degrees). (a) Transition energies as a function of applied stress in $\langle 111\rangle$ (left) and $\langle 110\rangle$ directions. Theoretical intensity of the $A^{(0)}$ transition is 0 : The line was not observed in experiment. (b) Temperature-dependent transition intensities at an applied $\langle 110\rangle$ stress of $1.3 \mathrm{GPa}$. Data given in $\pi$ (left) and $\sigma$ detection polarizations and have been normalized to the most intense transition.

interactions between the $E$ and $A$ are not taken into account within the model: High-quality absorption data under stress are required to confirm the presence of these interactions, and the low concentration of $\mathrm{SiV}^{0}$ in the present sample prohibits absorption measurements.

With the excited states' orbital degeneracy and symmetry under stress confirmed, we now reconcile our observations with the electronic model of $\mathrm{SiV}^{0}$. The EPR-active ${ }^{3} A_{2 g}$ ground state arises from the molecular orbital (MO) configuration $a_{1 g}^{2} a_{2 u}^{2} e_{u}^{4} e_{g}^{2}$ (三 $e_{g}^{2}$ in the hole picture, used henceforth), along with ${ }^{1} E_{g},{ }_{1} A_{1 g}$ [20]. The first one-electron excited state, $e_{u}^{1} e_{g}^{1}$ [19], generates ${ }^{1,3} A_{1 u},{ }^{1,3} A_{2 u}$, and ${ }^{1,3} E_{u}$ states, where the superscript 1,3 indicates that both spin-singlet and spin-triplet states are possible. As $e_{g}^{2}$ and $e_{u}^{1} e_{g}^{1}$ are the two lowest-energy one-electron configurations [20], we identify the doubly degenerate excited state observed under stress with the ${ }^{3} E_{u}\left(e_{u}^{1} e_{g}^{1}\right)$ state. The previous report assigning the 946-nm excited state to ${ }^{3} A_{1 u}$ [19] was based on a charge balance between $\mathrm{SiV}^{0}$ and $\mathrm{SiV}^{-}$, and did not account for the presence of $\mathrm{SiV}^{2-}[20,30]$, leading to an erroneous conclusion.

The requirement of applied stress for the observation of the non-orbitally-degenerate transitions (Fig. 1) indicates that the transitions are forbidden by orbital symmetry but not spin. As the only $S=1$ state arising from the $e_{g}^{2}$ configuration, we assume that the ground state of this transition is the EPR-active ${ }^{3} A_{2 g}$ : The singlet is then restricted by symmetry selection rules and the electronic model to ${ }^{3} A_{2 u}$. The observed $\Gamma_{2}^{-}$symmetry under stress matches this observation and hence we assign the symmetry ${ }^{3} A_{2 u}\left(e_{u}^{1} e_{g}^{1}\right)$. We identify this state with the shelving state observed in temperature-dependent PL measurements, where the intensity of the 946-nm ZPL was shown to decrease with decreasing temperature [19]. This conclusion is supported by recent $a b$ initio results which support the assignment of the levels given here [31]. We note that recent measurements on single centers do not appear to show the shelving effect [16]: It is unclear how to reconcile these observations with the present model.

In addition to the purely electronic transitions discussed above, the PL spectrum of $\mathrm{SiV}^{0}$ also exhibits a small feature at $976 \mathrm{~nm}$ [17]. In our measurements, we find that the energy shift of the transition under stress is essentially identical to the 946- and 951-nm transitions [Fig. 3(a)] [21]. As the line is at lower energy than the associated ZPLs, we identify it with a pseudo-LVM in the common ground state. This observation is incompatible with previous density functional theory (DFT) calculations suggesting that this transition is a stress-induced electronic transition between a ${ }^{3} E_{g}$ excited state and the ${ }^{3} A_{2 g}$ ground state [20].

To investigate the participation of $\mathrm{Si}$ in the pseudo-LVM, PL spectroscopy of a sample grown with isotopically enriched silicon dopant was performed: We find that the vibration frequency drops from $39.2 \mathrm{meV}$ in a natural abundance sample $\left(>90 \%{ }^{28} \mathrm{Si}\right.$ ) to $38.6 \mathrm{meV}$ in a sample enriched with $90 \%$ ${ }^{29} \mathrm{Si}$ [Fig. 3(b)]. Modeling the vibration as a simple harmonic oscillator, the mode frequency under isotopic enrichment is given by $\Omega^{*}=\Omega_{0} \sqrt{m^{*} / m_{0}}$, where $m^{*}$ is the effective mass of the isotopic enrichment, and $\Omega_{0}$ and $m_{0}$ are the mode frequency and effective mass in a natural abundance sample, respectively. Applying this model yields $\Omega_{\text {model }}^{*}=38.6 \mathrm{meV}$, matching the experimental value. This confirms that the LVM is primarily due to oscillation of the $\mathrm{Si}$ within the vacancy "cage," and is only weakly coupled to the bulk. Finally, the symmetry of the LVM may be addressed. The similar polarization behavior of the 946- and 976-nm transitions [Fig. 3(a)] indicates an $a_{1 g}$ mode. However, only $e_{u}$ or $a_{2 u}$ silicon oscillations participate in pseudo-LVM modes [32]: 

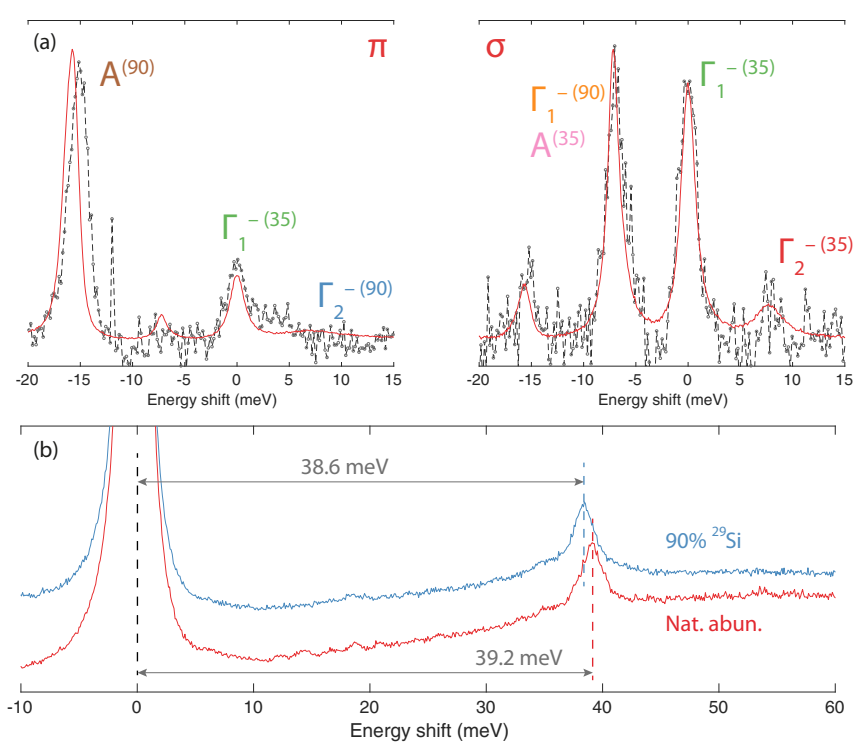

FIG. 3. (a) Comparison of PL measurements of the 946-nm and associated stress-induced transitions (solid lines) with the 976-nm local mode (dots). Measurements collected at $80 \mathrm{~K}$ with $\sigma_{\langle 110\rangle}=$ $2.1 \mathrm{GPa}$ for both $\pi$ (left) and $\sigma$ detection polarization. Individual transitions are labeled as in Fig. 2. (b) Effect of isotopic enrichment on the 976-nm local vibrational mode. The mode shifts from $\Omega_{0}=39.2 \mathrm{meV}$ in natural abundance material $\left(92 \%{ }^{28} \mathrm{Si}\right)$ to $\Omega^{*}=$ $38.6 \mathrm{meV}$ in a sample enriched with ${ }^{29} \mathrm{Si}$ (primary sample for this study). Treating the mode as a simple harmonic oscillation of the silicon atom yields $\Omega^{*}=38.6 \mathrm{meV}$, matching experiment. ZPLs have been fixed at zero for clarity.

In both these cases, the overall mode symmetry ${ }^{3} A_{2 g} \otimes \Gamma_{\mathrm{LVM}}$ becomes ungerade and thus vibronic transitions from both ${ }^{3} E_{u}$ and ${ }^{3} A_{2 u}$ excited states are forbidden by parity. We may reconcile the spectroscopic data with the model only by considering symmetry-lowering distortions. For example, under instantaneous symmetry-lowering distortions from $D_{3 d} \rightarrow$ $C_{3 v}$ due to (pseudo-)Jahn-Teller distortions in the excited state, the $a_{2 u}$ mode becomes $a_{1}$ and the vibronic transition is no longer forbidden. We observe no sharp mode related to the $e_{u}$ oscillation of the silicon. A similarly complex situation is encountered in $\mathrm{SiV}^{-}$, where two pseudo-LVMs have been identified at 40 and $64 \mathrm{meV}$ [26]. Studies of the latter indicate that its frequency is well approximated by a simple harmonic oscillator model [33] and essentially involves only the silicon atom, as we find for the $39-\mathrm{meV}$ mode of $\mathrm{SiV}^{0}$. However, experimental measurements assign the 64-meV mode to $a_{2 u}$ symmetry $[33,34]$ through polarized single-center studies, whereas recent hybrid-DFT calculations assign the mode $e_{u}$ symmetry and argue that the $40-\mathrm{meV}$ mode is not an LVM [32]. Further work is required to definitively identify the vibrational states of $\mathrm{SiV}$ in both charge states.

With knowledge of the excited-state symmetries and behavior under stress, we may reanalyze recent measurements of the spin polarization behavior [16,17]. The latter measurement identifies significant spin polarization at approximately $951 \mathrm{~nm}$ (Fig. S9 [16]): In light of our results on the stress-induced optical transition at $951 \mathrm{~nm}$, we understand that the measurement was performed on a strained ensemble,
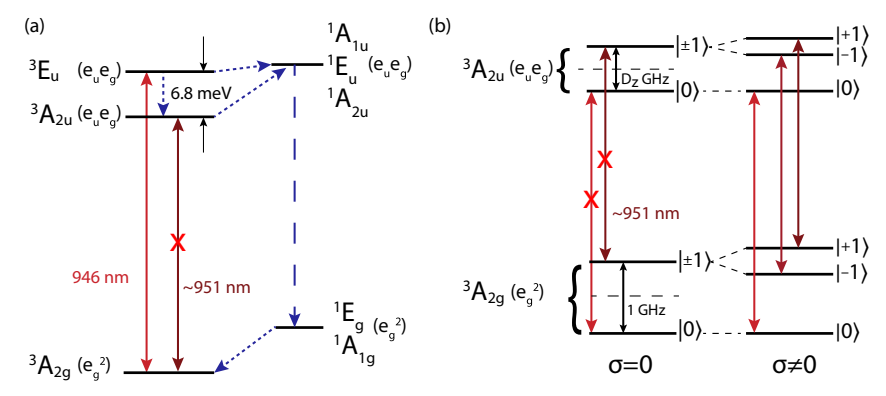

FIG. 4. (a) The electronic structure of $\mathrm{SiV}^{0}$ proposed as a result of uniaxial stress measurements. The ordering and relative energies of the spin singlets is not known. Electronic configurations are described in the hole picture, i.e., $e_{u} e_{g} \equiv a_{2 u}^{2} a_{1 g}^{2} e_{u}^{3} e_{g}^{3}$. (b) Proposed scheme for spin-dependent initialization and readout of the 951-nm transition under a small applied strain: $D_{z}$ is not known.

and interpret its visibility in an absorption spectrum as a direct transition from the ${ }^{3} A_{2 g}$ ground state to the ${ }^{3} A_{2 u}$ state [Fig. 4(a)]. As the measurements were completed by reading out spin polarization from the ${ }^{3} A_{2 g}$ ground state, and the temperature $(\approx 4 \mathrm{~K})$ was such that the thermal population of the ${ }^{3} E_{u}$ was negligible, this is direct evidence that the ${ }^{3} A_{2 u}$ excited state is involved in the spin polarization mechanism. The spin polarization mechanism when the ${ }^{3} E_{u}$ is excited must therefore either involve (a) both the ${ }^{3} E_{u}$ and ${ }^{3} A_{2 u}$ states, or (b) occur via an initial phonon relaxation from the ${ }^{3} E_{u}$ state to the ${ }^{3} A_{2 u}$ [Fig. 4(a)]. In the case of both (a) and (b), this level scheme enables the possibility of off-resonant optical spin readout and thus ODMR via the emission of the ${ }^{3} A_{2 u}$ state under stress: In (b) no ODMR is expected from the ${ }^{3} E_{u}$ state. Observation of ODMR would enable measurements of the intersystem-crossing rates for different excited-state electron spin states $\left(m_{S}=0, \pm 1\right)$, which, in addition to information on the relative ordering of the singlet states, is required for a full description of the spin polarization mechanism [21].

The thermal interaction of the ${ }^{3} E_{u}$ and ${ }^{3} A_{2 u}$ states poses a problem for the use of $\mathrm{SiV}^{0}$ as a photonic resource, as the intensity of the 946-nm transition decreases with decreasing temperature due to thermal depopulation from ${ }^{3} E_{u}$ into ${ }^{3} A_{2 u}$ : Typically, $<20 \mathrm{~K}$ is required to isolate spin-conserving optical transitions in diamond [35,36]. For small $(\lesssim 0.3$ GPA) stresses applied perpendicular to the symmetry axis, the intensity and frequency of the 951-nm transition is quadratic in stress: The stress will also remove the $m_{s}= \pm 1$ spin degeneracy in the spin triplets. Under stress, the spin-conserving optical transitions between the ${ }^{3} A_{2 g}$ ground state and ${ }^{3} A_{2 u}$ excited state are no longer forbidden [Fig. 4(b)], and in conjunction with the spin polarization mechanism in $\mathrm{SiV}^{0}$ may enable spin-dependent optical initialization and readout at low magnetic field. To optically readout the $m_{s}=0, \pm 1$ states we simply require the difference in the zero-field splitting of the ground state and excited state to be larger than the inhomogeneous linewidth of the transitions themselves, with high-fidelity initialization into $m_{s}=0$ completed via the spin polarization mechanism and coherent control between $0 \leftrightarrow$ +1 and $0 \leftrightarrow-1$ realized via coherent microwave control at nonzero magnetic field. All-optical control of the spin is enabled if the excited-state and ground-state spin manifolds 
possess different $g$-factors or spin-strain responses (or both). Implementation of this scheme would form the foundation of an $\mathrm{SiV}^{0}$ spin-photon interface [10].

The electronic structure of $\mathrm{SiV}^{0}$ requires further work to identify the internal spin polarization mechanism(s), and to identify the transitions predicted from this work. The present model is quantitatively sensitive to the precise numerical parameters used, but is nevertheless believed to be a qualitatively correct description of the first excited spin-triplet states. Future work should include monitoring strained $\mathrm{SiV}^{0}$ centers in both EPR and resonant PL to determine the effect of strain on the spin-spin interactions in both the orbital singlet states, and measurement of single centers under strain to identify spin-conserving optical transitions.

We thank B. G. Breeze at the University of Warwick Spectroscopy Research Technology Platform for helpful discussion and assistance with experiments. B.L.G. gratefully acknowledges the financial support of the Royal Academy of Engineering. This work is supported by EPSRC Grants No. EP/L015315/1 and No. EP/M013243/1, and ARC Grants No. DE170100169 and No. DP140103862.
[1] I. Aharonovich, D. Englund, and M. Toth, Nat. Photonics 10, 631 (2016).

[2] L. J. Rogers, K. D. Jahnke, T. Teraji, L. Marseglia, C. Müller, B. Naydenov, H. Schauffert, C. Kranz, J. Isoya, L. P. McGuinness, and F. Jelezko, Nat. Commun. 5, 4739 (2014).

[3] M. W. Doherty, N. B. Manson, P. Delaney, F. Jelezko, J. Wrachtrup, and L. C. L. Hollenberg, Phys. Rep. 528, 1 (2013).

[4] C. Hepp, T. Müller, V. Waselowski, J. N. Becker, B. Pingault, H. Sternschulte, D. Steinmüller-Nethl, A. Gali, J. R. Maze, M. Atatüre, and C. Becher, Phys. Rev. Lett. 112, 036405 (2014).

[5] T. Gaebel, M. Domhan, I. Popa, C. Wittmann, P. Neumann, F. Jelezko, J. R. Rabeau, N. Stavrias, A. D. Greentree, S. Prawer, J. Meijer, J. Twamley, P. R. Hemmer, and J. Wrachtrup, Nat. Phys. 2, 408 (2006).

[6] B. Hensen, H. Bernien, A. E. Dréau, A. Reiserer, N. Kalb, M. S. Blok, J. Ruitenberg, R. F. L. Vermeulen, R. N. Schouten, C. Abellán, W. Amaya, V. Pruneri, M. W. Mitchell, M. Markham, D. J. Twitchen, D. Elkouss, S. Wehner, T. H. Taminiau, and R. Hanson, Nature (London) 526, 682 (2015).

[7] P. Maletinsky, S. Hong, M. S. Grinolds, B. Hausmann, M. D. Lukin, R. L. Walsworth, M. Loncar, and A. Yacoby, Nat. Nanotechnol. 7, 320 (2012).

[8] Y. Wang, F. Dolde, J. Biamonte, R. Babbush, V. Bergholm, S. Yang, I. Jakobi, P. Neumann, A. Aspuru-Guzik, J. D. Whitfield, and J. Wrachtrup, ACS Nano 9, 7769 (2015).

[9] L. Rondin, J.-P. Tetienne, T. Hingant, J.-F. Roch, P. Maletinsky, and V. Jacques, Rep. Prog. Phys. 77, 056503 (2014).

[10] E. Togan, Y. Chu, A. S. Trifonov, L. Jiang, J. Maze, L. Childress, M. V. G. Dutt, a. S. Sørensen, P. R. Hemmer, A. S. Zibrov, and M. D. Lukin, Nature (London) 466, 730 (2010).

[11] H. Bernien, B. Hensen, W. Pfaff, G. Koolstra, M. S. Blok, L. Robledo, T. H. Taminiau, M. Markham, D. J. Twitchen, L. Childress, and R. Hanson, Nature (London) 497, 86 (2013).

[12] A. Sipahigil, R. E. Evans, D. D. Sukachev, M. J. Burek, J. Borregaard, M. K. Bhaskar, C. T. Nguyen, J. L. Pacheco, H. A. Atikian, C. Meuwly, R. M. Camacho, F. Jelezko, E. Bielejec, H. Park, M. Lončar, and M. D. Lukin, Science 354, 847 (2016).

[13] A. Sipahigil, K. D. Jahnke, L. J. Rogers, T. Teraji, J. Isoya, A. S. Zibrov, F. Jelezko, and M. D. Lukin, Phys. Rev. Lett. 113, 113602 (2014).

[14] S. Meesala, Y.-I. Sohn, B. Pingault, L. Shao, H. A. Atikian, J. Holzgrafe, M. Gundogan, C. Stavrakas, A. Sipahigil, C. Chia, M. J. Burek, M. Zhang, J. L. Pacheco, J. Abraham, E. Bielejec,
M. D. Lukin, M. Atature, and M. Loncar, Phys. Rev. B 97, 205444 (2018).

[15] D. D. Sukachev, A. Sipahigil, C. T. Nguyen, M. K. Bhaskar, R. E. Evans, F. Jelezko, and M. D. Lukin, Phys. Rev. Lett. 119, 223602 (2017).

[16] B. C. Rose, D. Huang, Z.-H. Zhang, P. Stevenson, A. M. Tyryshkin, S. Sangtawesin, S. Srinivasan, L. Loudin, M. L. Markham, A. M. Edmonds, D. J. Twitchen, S. A. Lyon, and N. P. de Leon, Science 361, 60 (2018).

[17] B. L. Green, S. Mottishaw, B. G. Breeze, A. M. Edmonds, U. F. S. D'Haenens-Johansson, M. W. Doherty, S. D. Williams, D. J. Twitchen, and M. E. Newton, Phys. Rev. Lett. 119, 096402 (2017).

[18] A. M. Edmonds, M. E. Newton, P. M. Martineau, D. J. Twitchen, and S. D. Williams, Phys. Rev. B 77, 245205 (2008).

[19] U. F. S. D’Haenens-Johansson, A. M. Edmonds, B. L. Green, M. E. Newton, G. Davies, P. M. Martineau, R. U. A. Khan, and D. J. Twitchen, Phys. Rev. B 84, 245208 (2011).

[20] A. Gali and J. R. Maze, Phys. Rev. B 88, 235205 (2013).

[21] See Supplemental Material at http://link.aps.org/supplemental/ 10.1103/PhysRevB.99.161112 for a description of the growth conditions, experimental geometry and apparatus, comparison of spectra with different input polarizations, the complete model and derivation of the analytical solutions to the coupled stress Hamiltonian, the model parameters used to generate the simulation, details on the computation of transition intensities, and notes on the spin polarization mechanism.

[22] L. Allers and A. T. Collins, J. Appl. Phys. 77, 3879 (1995).

[23] A. E. Hughes and W. A. Runciman, Proc. Phys. Soc. 90, 827 (1967).

[24] G. Davies and M. E. R. Hamer, Proc. R. Soc. London, Ser. A 348, 285 (1976).

[25] L. J. Rogers, M. W. Doherty, M. S. J. Barson, S. Onoda, T. Ohshima, and N. B. Manson, New J. Phys. 17, 013048 (2015).

[26] L. J. Rogers, K. D. Jahnke, M. W. Doherty, A. Dietrich, L. P. McGuinness, C. Müller, T. Teraji, H. Sumiya, J. Isoya, N. B. Manson, and F. Jelezko, Phys. Rev. B 89, 235101 (2014).

[27] Y. N. Palyanov, I. N. Kupriyanov, Y. M. Borzdov, and N. V. Surovtsev, Sci. Rep. 5, 14789 (2015).

[28] P. Delaney, J. C. Greer, and J. A. Larsson, Nano Lett. 10, 610 (2010).

[29] G. Davies, J. Phys. C 12, 2551 (1979). 
[30] S. Häußler, G. Thiering, A. Dietrich, N. Waasem, T. Teraji, J. Isoya, T. Iwasaki, M. Hatano, F. Jelezko, A. Gali, and A. Kubanek, New J. Phys. 19, 063036 (2017).

[31] G. Thiering and A. Gali, npj Comput. Mater. 5, 18 (2019).

[32] E. Londero, G. Thiering, L. Razinkovas, A. Gali, and A. Alkauskas, Phys. Rev. B 98, 035306 (2018).

[33] A. Dietrich, K. D. Jahnke, J. M. Binder, T. Teraji, J. Isoya, L. J. Rogers, and F. Jelezko, New J. Phys. 16, 113019 (2014).
[34] L. J. Rogers, K. D. Jahnke, M. H. Metsch, A. Sipahigil, J. M. Binder, T. Teraji, H. Sumiya, J. Isoya, M. D. Lukin, P. Hemmer, and F. Jelezko, Phys. Rev. Lett. 113, 263602 (2014).

[35] K.-M. C. Fu, C. Santori, P. E. Barclay, L. J. Rogers, N. B. Manson, and R. G. Beausoleil, Phys. Rev. Lett. 103, 256404 (2009).

[36] L. Nicolas, T. Delord, P. Huillery, E. Neu, and G. Hétet, AIP Adv. 8, 065102 (2018). 